\title{
Efecto de temperatura y velocidad de secado en la actividad antioxidante de la mashua (Tropaeolum tuberosum) en el secado en bandejas
}

\section{Effect of temperature and drying speed on the antioxidant activity of mashua (Tropaeolum tuberosum) in tray drying}

\author{
Royler Alvis Celis ${ }^{1}$
}

\section{RESUMEN}

La investigación evaluó el efecto de temperatura y velocidad de secado en la actividad antioxidante de la mashua (T. tuberosum) en el secado en bandejas, debido a que la pérdida de actividad antioxidante, durante el procesado de los alimentos se ha vuelto un problema, debido a su baja resistencia a temperaturas altas de secado . Se trabajó con mashua amarilla procedente de la provincia de Luya, ubicada a una altitud de $2311 \mathrm{msnm}$. En el presente estudio, se realizó un experimento factorial 3Ax3B bajo un Diseño Completamente al Azar con tres repeticiones, donde se emplearon diferentes temperaturas y velocidad de aire. Se determinó el efecto de la temperatura de secado, la velocidad de aire, y la interacción de ambos no es significativo en el porcentaje de captaciones de radicales libres, puesto que el valor crítico para $\mathrm{F}$, en todos los casos es mayor a 0,05 . Se logró una capacitación de radicales libres mayor al 50\%, demostrando su potencial capacidad antioxidante de la mashua.

Palabras clave: Mashua, capacidad antioxidante, secado.

\begin{abstract}
The investigation evaluated the effect of temperature and speed of drying in the antioxidant activity of the mashua (T. tuberosum) in the drying in trays, because the loss of antioxidant activity, during the processing of food has become a problem, due to its low resistance to high drying temperatures (Cuya, 2009). We worked with yellow mashua from the province of Luya, located at an altitude of 2311 meters above sea level. In the present study, a 3Ax3B factorial experiment was carried out under a Completely Random Design with three repetitions, where different temperatures and air velocity were used. The effect of the drying temperature, the air velocity, and the interaction of both was not significant in the percentage of free radical uptake, since the critical value for $\mathrm{F}$, in all cases is greater than 0.05 . A free radical training of more than $50 \%$ was achieved, demonstrating its potential antioxidant capacity of the mashua.
\end{abstract}

Keywords: Mashua, antioxidant capacity, drying.

${ }^{1}$ Bach. Ingeniería Agroindustrial. Universidad Nacional Toribio Rodríguez de Mendoza de Amazonas. Correo electrónico: roylerac24@hotmail.com 


\section{INTRODUCCIÓN}

Durante los últimos años la comunidad científica orientada a la alimentación, viene enfocándose dentro de muchas cosas en alimentos funcionales. Una de las propiedades funcionales más importantes de una matriz alimentaria es su capacidad antioxidante, puesto que se ha comprobado que son los responsables de tratamientos y prevención de algunas patologías, como también la conservación de alimentos. Dada su composición, las moléculas antioxidante evitan el deterioro de las grasas y otras moléculas con alta susceptibilidad de oxidarse (Chan, 2015).

Junto a muchas matrices alimentarias provenientes o no de la agricultura, en el Perú se han estudiado las moléculas responsables de la acción antioxidante y se ha encontrado que lo compuestos fenólicos son los principales antioxidantes presentes en los vegetales. La mashua (Tropaeolum tuberosum Ruiz \& Pavón) es un tubérculo rico en compuestos fenólicos, antociánicos y carotenoides , comparable incluso al arándano, fruta antioxidante por excelencia excelente (Lara,2017).

Un estudio comparativo, reporta que T. tuberosum posee actividad antioxidante superior incluso a otros tubérculos andinos muy consumidos por su calidad nutricional (Campos et al., 2016)

Mejía et al. (2018) ha encontrado que todas las partes de la planta de T. tuberosum posee una elevada actividad antioxidante, asimismo menciona que su capacidad es más alto o comparable a las fuentes conocidas de antioxidantes naturales.

La industrialización de éste tubérculo ha sido también estudiado y debido a que el objetivo de los procesos de transformación es procesar buscando causar el mínimo impacto en la calidad nutritiva de los alimentos; se ha evidenciado que la temperatura y velocidad del aire en un proceso de deshidratación son fundamentales (Lara, 2017) y que antes de proponer una técnica, debe definirse adecuadamente éstos parámetros con la finalidad de obtener un deshidratado con la mayor capacidad antioxidante posible.

También, debe tenerse en cuenta la variedad de $T$. tuberosum con la que se está trabajando, puesto que estudios anteriores han evidenciado que además de poseer diferentes compuestos responsables de su actividad antioxidante, éstos requieren de diferentes condiciones de secado (Chirinos et al., 2007; Taipe, 2017). Se ha relacionado la actividad antioxidante con el color de los tubérculos, los de color morado están asociados a la elevada presencia de compuestos antociánicos y los de color amarillo a compuestos fenólicos y carotenoides (Chirinos et al., 2008;
Páucar, 2014).

Como complemento a lo antes descrito Inostroza et al. (2015) y Saavedra (2017) mencionan que los pigmentos presentes del tubérculo de T. tuberosum, de piel y pulpa morada, poseen una importante actividad antioxidante, sin embargo, las variedades de otro color también deben estudiarse puesto que tienen otros compuestos con elevada capacidad antioxidante.

Debe tenerse en cuenta además que los alimentos deshidratados tienen muchas ventajas, ya que al reducir el contenido de humedad y de la actividad de agua se logra inactivar y controlar el crecimiento de microorganismos patógenos y en descomposición, asegurando alimentos estables y saludables (Húngaro et al., 2014) También el secado de los alimentos reduce su peso y en muchos casos el volumen, lo que incluye en una reducción importante, de los costos de empaque, almacenamiento y transporte. Los productos secos además permiten ser almacenados a temperatura ambiente por largos periodos de tiempo (Badui, 2012).

En consecuencia, el objetivo de investigación fue evaluar el efecto de temperatura y velocidad de secado en la actividad antioxidante de $T$. tuberosum en el secado en bandejas.

\section{MATERIAL Y MÉTODOS}

\section{Obtención del material de estudio}

Se trabajó con mashua amarilla procedente del Distrito de Conila de la Provincia de Luya, Región Amazonas, ubicada a una altitud de $2311 \mathrm{msnm}$.

Las muestras fueron recolectadas en el campo y trasladadas a laboratorio dentro los 8 días después de la cosecha; fueron lavados con agua, secadas al ambiente.

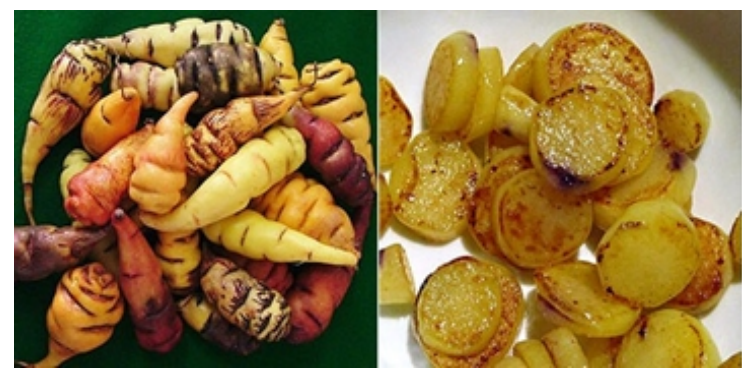

Figura 1. Tubérculos de T. tuberosum seleccionados para el estudio.

\section{Diseño experimental}

En el presente estudio, se realizó un experimento factorial 3Ax3B bajo un Diseño Completamente al Azar (DCA) con tres repeticiones (tabla 1). 
Tabla 1. Arreglo del experimento

\begin{tabular}{|c|c|c|c|c|c|c|c|c|c|}
\hline $\begin{array}{l}\text { Temperatura } \\
\text { de secado }\end{array}$ & \multicolumn{3}{|c|}{40} & \multicolumn{3}{|c|}{50} & \multicolumn{3}{|c|}{60} \\
\hline $\begin{array}{l}\text { Velocidad de } \\
\text { aire }(\mathrm{m} / \mathrm{s})\end{array}$ & 2 & 3 & 5 & 2 & 3 & 5 & 2 & 3 & 5 \\
\hline Tratamientos & $\mathrm{T} 1$ & $\mathrm{~T} 2$ & $\mathrm{~T} 3$ & $\mathrm{~T} 4$ & T5 & T6 & $\mathrm{T} 7$ & T8 & T9 \\
\hline \multirow{3}{*}{ Repeticiones } & R1 & R1 & R1 & R1 & R1 & R1 & R1 & R1 & R1 \\
\hline & $\mathrm{R} 2$ & $\mathrm{R} 2$ & R2 & $\mathrm{R} 2$ & R2 & $\mathrm{R} 2$ & R2 & $\mathrm{R} 2$ & $\mathrm{R} 2$ \\
\hline & R3 & R3 & R3 & R3 & R3 & R3 & R3 & R3 & R3 \\
\hline
\end{tabular}

\section{Procedimiento}

\section{Secado de mashua}

Los tubérculos seleccionados se hizo cortes en rodajas ( $2 \mathrm{~mm}$ aproximadamente). Para el estudio correspondiente, se emplearon temperaturas de secado teniendo en cuenta tres niveles de 40, 50 y 60 ${ }^{\circ} \mathrm{C}$ y velocidades de aire con niveles de 2,3 y $5 \mathrm{~m} / \mathrm{s}$, donde se aplicó a cada uno de los tratamientos.

Después de obtener las rodajas secas, se obtuvo harina con la ayuda de una máquina de moler tradicional, luego fue colocada en un tamiz vibratorio para obtener así una harina más fina y uniforme. Luego todas las muestras fueron analizadas mediante el método del DPPH.

\section{Determinación de la actividad antioxidante:}

Para esta se recurrió a la técnica de Brand-Williams et al. (1995), pero modificado para la presente investigación. Consistió en:

Se preparó $400 \mathrm{ml}$ de una solución de DPPH $(1,1-$ diphenil-2-picril hidracilo) en metanol de $75 \mathrm{mg} / \mathrm{L}$; luego se prepararon soluciones metanólicas de la muestra en concentraciones de $900 \mu \mathrm{g} / \mathrm{ml}$ (solución A).

Se realizó el ajuste del espectrofotómetro digital UNICO modelo S-2100 a cero, con un blanco de metanol agua $2: 1$; posteriormente se preparó el blanco de muestra con $0,75 \mathrm{ml}$ de muestra (solución A) y 1,5 ml de metanol; así también se preparó el patrón de referencia con 1,5 $\mathrm{ml}$ de solución de DPPH y $0,75 \mathrm{ml}$ de agua. Luego preparamos las muestras con $0,75 \mathrm{ml}$ de solución A y 1,5 ml de solución de DPPH, dejamos actuar por $5 \mathrm{~min}$ y se realiza la lectura a $517 \mathrm{~nm}$ en el espectrofotómetro.

Se realizó la lectura de la absorbancia del patrón de referencia y del blanco de muestra, seguidamente se tomó las lecturas de las 9 muestras preparadas; con los valores de las absorbancias obtenidas se determinó el \% de captación de radicales libres (DPPH) mediante la siguiente formula:

\% captación de radical libre:

$[1-(\mathrm{A} 2-\mathrm{A} 3) / \mathrm{A} 1]^{*} 100$

Dónde:

A1=Absorbancia del patrón de referencia;

$\mathrm{A} 2=$ Absorbancia de la muestra;

A3 = Absorbancia del blanco de muestra

\section{RESULTADOS}

La mashua amarilla, proveniente de la provincia de Luya, presentó un porcentaje de captaciones de radicales libres entre 90,86 a 97,94\%, correspondiente al tratamiento $\mathrm{T} 3 \mathrm{y}$ al tratamiento $\mathrm{T} 7$, respectivamente.

Tabla 2. Porcentaje de captaciones de radicales libres para los tratamientos.

\begin{tabular}{c|c|c}
\hline Tratamiento & $\mathbf{T}^{\circ} \mathbf{C} / \mathbf{v e l o c i d a d}$ aire (m/s) & \% Captación de radicales libres \\
& & \\
\hline T1 & $40-2$ & 96,40 \\
\hline T2 & $40-3$ & 95,28 \\
\hline T3 & $40-5$ & 90,86 \\
\hline T4 & $50-2$ & 95,87 \\
\hline T5 & $50-3$ & 92,63 \\
\hline T6 & $50-5$ & 96,76 \\
\hline T7 & $60-2$ & 97,94 \\
\hline T8 & $60-3$ & 95,58 \\
\hline T9 & $60-5$ & 94,99 \\
\hline
\end{tabular}

Ninguno de los tratamientos tuvo efecto en la actividad antioxidante de mashua tal como se evidencia en la tabla 3 (sig: 0,05). 
Tabla 3. ANOVA de los tratamientos empleados

\begin{tabular}{ccccccc}
\hline $\begin{array}{c}\text { Origen de las } \\
\text { variaciones }\end{array}$ & $\begin{array}{c}\text { Suma de } \\
\text { cuadrados }\end{array}$ & $\begin{array}{c}\text { Grados } \\
\text { de } \\
\text { libertad }\end{array}$ & $\begin{array}{c}\text { Promedio } \\
\text { de los } \\
\text { cuadrados }\end{array}$ & F & Probabilidad & $\begin{array}{c}\text { Valor } \\
\text { crítico } \\
\text { para F }\end{array}$ \\
\hline Temperatura & 0,0025 & 2 & 0,00125 & 2,160 & 0,1443 & 3,5546 \\
\hline $\begin{array}{c}\text { Velocidad de } \\
\text { viento }\end{array}$ & 0,0011 & 2 & 0,00057 & 0,977 & 0,3954 & 3,5546 \\
\hline $\begin{array}{c}\text { Interacción } \\
\text { Dentro del }\end{array}$ & 0,0024 & 4 & 0,00060 & 1,034 & 0,4170 & 2,9277 \\
grupo & 0,0104 & 18 & 0,00058 & & & \\
\hline Total & 0,0165 & 26 & & & & \\
\hline
\end{tabular}

La Tabla 5, referente a la velocidad de pérdida de peso en la mashua durante la etapa de secado y su efecto sobre la capacidad antioxidante demuestra que no existe diferencia significativa entre este indicador y la velocidad de secado.

Aunque las diferencias no son significativas (Tabla 3 ), en la figura 3 se observa que las muestras secadas a mayor temperatura tuvieron mayor actividad antioxidante.

En cuanto a la velocidad del aire, a mediad que se incrementa la velocidad del aire de secado, se reduce la actividad antioxidante.

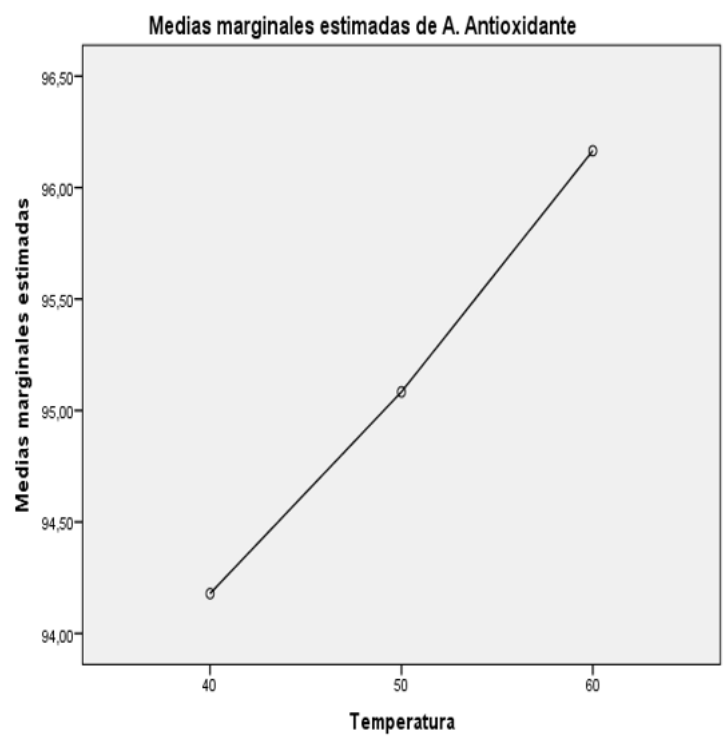

Figura 2. Actividad antioxidante de mashua secada a diferentes temperaturas

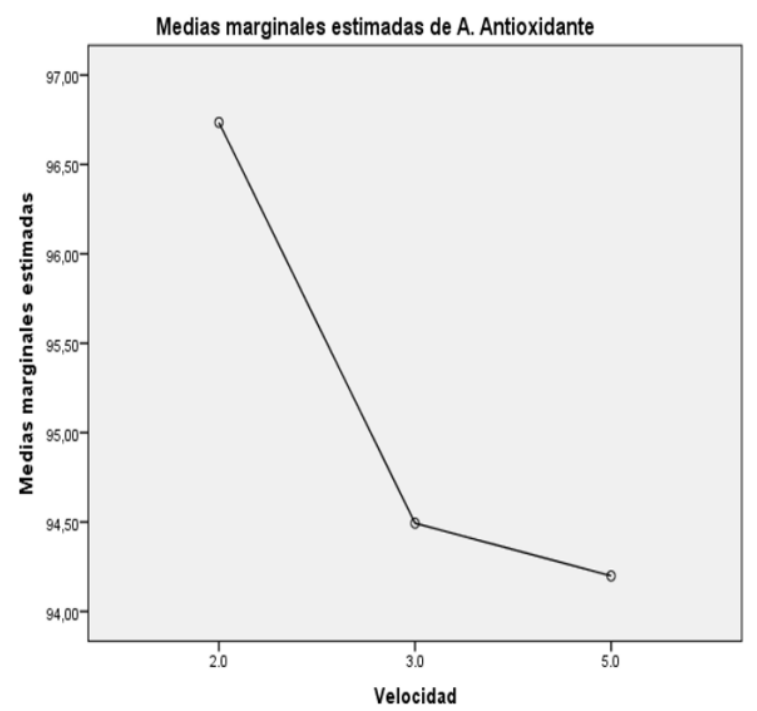

Figura 3. Actividad antioxidante según las velocidades.

\section{DISCUSIÓN}

El maximo valor de captación de radicales libres $(97,94 \%)$ se dio a $60^{\circ} \mathrm{C}-2 \mathrm{~m} / \mathrm{s}$ y el menor valor encontrado $(90,86 \%)$ fue con condiciones de secado de $40^{\circ} \mathrm{C}-5 \mathrm{~m} / \mathrm{s}$, observándose una variación de aproximadamente el 7\%. Esta variación en el valor, probablemente se debe a las reacciones de oxidanción durande el secado, por reacción directamente con el oxígeno o otros componentes, y con menor probabilidad con los radicales de los lípidos, principalemnte hidroperóxidos (compuestos que tambien reaccionan con los compuestos antioxidantes) es baja debido al corto tiempo(Chou y Chua, 2001), ademas porque el contenido de grasa en la mashua es bajo ( $1 \%$ ). Estas reacciones se aceleran con las altas temperaturas, disminuyendo la capacidad antioxidante. Lo evidenciado en la presente investigación se corrobora con lo que 
menciona Pacco (2015), quien determinó que a medida que sube la temperatura de cocción disminuye la capacidad antioxidante (por encima de los $60^{\circ} \mathrm{C}$ como referencia)

Aunque la diferencia entre los tratamientos no fue estadísticamente significactivo, se observa que a medida que se incrementaba la velocidad del aire de secado, la actvidad antioxidante se redujo (Figura 4); lo cual podría ser indicador de que los compuestos presentes en T. tuberosum, principalmente fenoles y carotenos, son termolábiles(Chirinos, Rogez, Campos, Pedreschi, \& Larondelle, 2007).

Según la teoría, a menores temperaturas de secado se modifican menos los compuestos de la matriz y se obtienen deshidratados mejor conservados y con mayor capacidad antioxidante; sin embargo, tal como se evidencia en la Figura 3, a medida que se incrementó la temperatura de secado, se obtuvo deshidratados con mayor actividad antioxidante; al respecto Cuya (2009) observado que la mejor retención de compuestos con capacidad antioxidante en T. tuberosum se obtiene al deshidratarla entre $50 \mathrm{y}$ $60{ }^{\circ} \mathrm{C}$; podría deberse a que se incrementa la concentración o el proceso influye en la generación de nuevos componentes.

Los valores de actividad antioxidante (figura 3) obtenidos son relativamente elevados, indicador de que los rangos de temperatura y velocidad del aire de secado han sido los adecuados, Palacios (2013) obtuvo resultados similares, por lo que se confirma éstos parámetros como óptimos para deshidratar $T$. tuberosum en secador de bandejas y garantizar un producto con potencialidad alimentaria de calidad (Campos, Noratto, Chirinos, Arbizu, \& Roca, 2006).

Aunque no se han evidenciado diferencias significativas en los tratamientos, los resultados evidencian que la mashua es una matriz muy estable durante el secado, sus propiedades antioxidantes no se ven modificadas ni por la temperatura ni por la velocidad del aire. No obstante, de acuerdo a la literatura, si estos parámetros toman valores extremos podría obtenerse resultados adversos (Chirinos, Rogez, Campos, Pedreschi, \& Larondelle, 2007).

\section{CONCLUSIONES}

Se concluye según el análisis estadístico que la temperatura de secado, la velocidad del viento y la interacción de los dos, no es significativa en el porcentaje de captaciones de radicales libres (alcanzando un valor mayor de $97,94 \%$ a $60^{\circ} \mathrm{C}$ y 2 $\mathrm{m} / \mathrm{s})$.

La actividad antioxidante de mashua no sufre variaciones considerables durante el secado, por lo que la temperatura del equipo puede oscilar entre 40 y $60{ }^{\circ} \mathrm{C}$ y la velocidad 2 y $5 \mathrm{~m} / \mathrm{s}$.
Asimismo, de los tratamientos aplicados se determinó que con las temperaturas trabajadas se logra una capacitación de radicales libres mayor al $50 \%$, demostrando su potencial capacidad antioxidante de la mashua.

Del mismo modo, como no existe diferencias significativas en todos los tratamientos se escogería por conveniencia cualquiera de ellos, ahorrando así energía durante el secado ya que al seleccionar la menor temperatura estaríamos ahorrando energía, así también por otro lado se ahorraría tiempo en el secado, seleccionando la mayor temperatura de los tratamientos.

\section{REFERENCIAS BIBLIOGRÁFICAS}

Badui, S. (2012). La ciencia de los alimentos en la práctica (Primera). México: Pearson. $\mathrm{R}$ e c u p e r a d o d e https://datospdf.com/download/la-cienciade-los-alimentos-en-la-practica_5a44d6f1b7d7bc422b92cedf_pdf

Brand-Williams, W., Cuvelier, M., \& Berset, C. (1995). Use of a Free Radical Method to Evaluate Antioxidant Activity. Lebensm. Wiss. Technol., 28(1), 25-30.

Campos, D., Noratto, G., Chirinos, R., Arbizu, C., Roca, W., \& Cisneros-Zevallos, L. (2006). Antioxidant capacity and secondary metabolites in four species of Andean tuber crops: native potato (Solanum sp.), mashua (Tropaeolum tuberosum Ruiz \& Pavón), Oca (Oxalis tuberosa Molina) and ulluco (Ullucus tuberosus Caldas). Journal of the Science of Food and Agriculture, 86(10), 1481-1488. https://doi.org/10.1002/jsfa.2529

Canahualpa Carhuamaca, F., \& Solano Gomez, E. (14 de Setiembre de 2018). Estudio de la cinética de secado y degradación de las antocianinas y vitamina $c$ de la mashua negra (Tropaeolum tuberosum). Obtenido de http://repositorio.uncp.edu.pe/handle/UNCP 14369

Chan, J. P. (2015). Eficacia antioxidante de los compuestos fenólicos de la mashua (Tropaeolum tuberosum) en la estabilidad del aceite de linaza (Linum usitatissimum L.) (Tesis de Grado). Universidad Nacional Agraria La Molina, Lima, Perú. Recuperado d

http://repositorio.lamolina.edu.pe/handle/U NALM/1791

Chirinos, R., Campos, D., Arbizu, C., Rogez, H., Rees, J.-F., Larondelle, Y., ... CisnerosZevallos, L. (2007). Effect of genotype, 
maturity stage and post-harvest storage on phenolic compounds, carotenoid content and antioxidant capacity, of Andean mashua tubers (Tropaeolum tuberosum Ruiz \& Pavón). Journal of the Science of Food and Agriculture, 87(3), $437-446$. https://doi.org/10.1002/jsfa.2719

Chirinos, R., Campos, D., Costa, N., Arbizu, C., Pedreschi, R., \& Larondelle, Y. (2008). Phenolic profiles of andean mashua (Tropaeolum tuberosum Ruíz \& Pavón) tubers: Identification by HPLC-DAD and evaluation of their antioxidant activity. Food Chemistry, $106(3), 1285-1298$. https://doi.org/10.1016/j.foodchem.2007.07 .024

Chou, S. K., \& Chua, K. J. (2001). New hybrid drying technologies for heat sensitive foodstuffs. Trends in Food Science \& Technology, $12\left(\begin{array}{ll}10\end{array}\right), \quad 359-369$. https://doi.org/10.1016/S09242244(01)00102-9

Cuya, R. A. (2009). Efecto de secado en bandeja y atomización sobre la actividad antioxidante de la mashua (Tropaeolum tuberosum $R \& P$ ) (Tesis de Maestría). Universidad Nacional Agraria La Molina, Lima, Perú. Recuperado d http://repositorio.lamolina.edu.pe/handle/U NALM/1703

Húngaro, H. M., Peña, W. E. L., Silva, N. B. M., Carvalho, R. V., Alvarenga, V. O., \& Sant'Ana, A. S. (2014). Food Microbiology. En Encyclopedia of Agriculture and Food Systems (pp. 213-231). Brasil: Elsevier. https://doi.org/10.1016/B978-0-444-525123.00059-0

Inostroza, L. A., Castro, A. J., Hernández, E. M., Carhuapoma, M., Yuli, R. A., Collado, A., \& Córdova, J. S. (2015). Actividad antioxidante de Tropaeolum tuberosum Ruiz \& Pavón (Mashua) y su aplicación como colorante para yogur. Ciencia e Investigación, 18(2), 83-89.

Lara, M. V. (2017). Deshidratación de Mashua Tropaeolum tuberosum para la obtención de hojuelas (Tesis de Grado). Universidad Técnica del Norte, Ibarra, Ecuador. $\mathrm{R}$ e c u p e r a d o d e http://repositorio.utn.edu.ec/handle/123456 $789 / 6153$

Mejía, F. M., Salcedo, J. E., Vargas, S., Serna, J. A., \& Torres, L. S. (2018). Capacidad antioxidante $y$ antimicrobiana de tubérculos andinos (Tropaeolum tuberosum y Ullucus tuberosus). Revista U.D.C.A Actualidad \& Divulgación Científica, 21(2), 449-456. https://doi.org/10.31910/rudca.v21.n2.2018 .1083

Pacco, W. (2015). Evaluación del efecto del soleado y la cocción en la capacidad antioxidante del puré deshidratado de Mashua (T. tuberosum r. et p.) (Tesis de Grado). Universidad Nacional del Altiplano, Puno. Recuperado de http://repositorio.unap.edu.pe/handle/UNAP $/ 2415$

Palacios, M. (2013). Influencia de blanqueado y secado a dos temperaturas en el contenido de compuestos fenolicos, carotenoides y capacidad antioxidante de los tubérculos de mashua (Tropaeolum Tuberosum Ruiz \& Pavón) (Tesis de Grado). Universidad Nacional de San Martín, Tarapoto, Perú. $\mathrm{R}$ e c u p e r a d o d e http://repositorio.unsm.edu.pe/bitstream/han dle/UNSM/526/TFAI_13.pdf?sequence $=1 \&$ isAllowed $=\mathrm{y}$

Paucar, S. A. (2014). Composición química y capacidad antioxidante de dos variedades de mashua (Tropaeolum tuberosum): amarilla chaucha y zapallo (Tesis de Grado). Universidad Tecnológica Equinoccial, Quito, E c u a d o r. R e c u p e r a d o d e http://repositorio.ute.edu.ec/bitstream/1234 56789/5112/1/58311_1.pdf

Saavedra, J. del C. (2017). Microencapsulación y su efecto en la retención de la capacidad antioxidante de la mashua (Tropaeolum tuberosum) secada por atomización Lambayeque (Tesis de Grado). Universidad Señor de Sipán, Lambayeque. Recuperado de http://repositorio.uss.edu.pe/bitstream/handl $\mathrm{e} / \mathrm{u} \mathrm{s} \mathrm{s} / 4113 / \mathrm{S}$ a a ved r a $\% 20$ $\% 20 \mathrm{~T} \% \mathrm{C} 3 \% \mathrm{~A} 1$ vara.pdf? sequence=1\&isAll owed $=\mathrm{y}$

Taipe, L. (2017). Fenoles totales y actividad antioxidante en mashua (Tropaeolum tuberosum) en estado fresco, soleado y cocido de las variedades amarillo zapallo y negra (Tesis de Grado). Universidad Nacional del Centro del Perú, Huancayo, $\mathrm{P}$ e r ú . R e c u p e r a d o d e http://repositorio.uncp.edu.pe/handle/UNCP $/ 1592$ 